EPJ manuscript No.

(will be inserted by the editor)

\title{
Dynamics of multi-frequency minority games
}

\author{
Andrea De Martino \\ INFM-SMC and Dipartimento di Fisica, Università di Roma "La Sapienza", p.le A. Moro 2, 00185 Roma (Italy) \\ e-mail: andrea.demartino@roma1.infn.it
}

\begin{abstract}
The dynamics of minority games with agents trading on different time scales is studied via dynamical mean-field theory. We analyze the case where the agents' decision-making process is deterministic and its stochastic generalization with finite heterogeneous learning rates. In each case, we characterize the macroscopic properties of the steady states resulting from different frequency and learning rate distributions and calculate the corresponding phase diagrams. Finally, the different roles played by regular and occasional traders, as well as their impact on the system's global efficiency, are discussed.
\end{abstract}

PACS. 87.23.Ge Dynamics of social systems - 05.65.+b Self-organized systems - 02.50.Le Decision theory and game theory - 05.10.Gg Stochastic analysis methods

\section{Introduction}

The collective phenomena that characterize the evolution of competitive populations of adaptive agents, such as the onset of cooperation or the creation of exploitable information, have attracted a great deal of attention from statistical physicists over the past few years. The hope is that the occurrence of such macroscopic effects can be understood starting from the laws that govern the behavior of the individual agents. Thanks to the basic simplicity of its definition, the minority game (MG) $[1,2]$ allows a full theoretical analysis of many of these issues. Originally, it ' was designed to mimic a market of speculators subject to the law of supply and demand. At each time step, traders react to the receipt of a public information pattern (the ' 'state of the world') by either buying or selling, trying to profit from price fluctuations to maximize their payoff, and learning from experience. Upon decreasing the relative number $\alpha$ of possible information patterns, the degree of cooperation in the system, measured by the inverse magnitude of global fluctuations, increases, until a critical point $\alpha_{c}$ is reached, below which highly cooperative as well as highly uncooperative states can occur, depending on the initial conditions of the agents' learning processes. Remarkably, the low $\alpha$ phase is characterized by the absence of exploitable information, while in the high $\alpha$ phase the market is to some degree predictable and hence inefficient.

On the technical level, the MG is defined by a set of globally coupled zero-temperature Markov processes with quenched disorder and without detailed balance. The system at $\alpha_{c}$ undergoes a dynamical phase transition with ergodicity breaking, related to the onset of anomalous response. The stationary state is solvable, at least in the ergodic phase, via both static (replica) and dynamical methods, so that a very deep understanding of its macroscopic properties is achievable. Moreover, the MG is open to a large class of economically- and biologically-inspired extensions, that allow to tackle such key issues as the interplay of different types of traders in a market [2].

An interesting modification of the original model is obtained when synchronicity is removed, and one accounts for the possibility that agents trade on different time scales. This is the 'colored' minority game (CMG) first introduced in [3]. Assuming a power-law distribution of trading frequencies and using the static approach of $[4,5]$, it was shown that, while the phase transition picture is substantially preserved, the actual critical point $\alpha_{c}$ depends on the particular exponent entering the power law. Furthermore, regular and occasional traders were shown to take markedly different behaviors, with the latter less prone to change trading strategy and hence contributing significantly less to the growth of global fluctuations.

In this work, we adapt the generating functional methods originally devised for spin glasses [6-10] and already employed in the theory of MGs [11-15] to study the dynamics of the CMG in a few different cases. First, assuming that the agents' learning process is deterministic, we derive a stochastic equation describing the behavior of an effective agent trading with frequency $f$ (Sec. 2). Global dynamical quantities such as correlation and response functions appear as proper averages over the trading frequency distribution $q(f)$. We calculate the phase diagrams exactly and evaluate macroscopic order parameters in ergodic stationary states explicitly in the simple case where $q(f)$ is bimodal (i.e. for a mixed population of frequent and occasional traders, with a wide separation of time scales between the former and the latter, Sec. 3) and when it has a power-law form (i.e. when there is no sin- 
Andrea De Martino: Dynamics of multi-frequency minority games

gle characteristic time scale for trading, Sec. 4). The roles of regulars and irregulars can be thoroughly investigated. Then (Sec. 5), we move to the case where the agents' learning process is stochastic by introducing different learning rates as a further element of heterogeneity among agents (besides their strategies and trading frequencies). We analyze additive and multiplicative decision noise (Sec. 6), deriving the corresponding phase structure as a function of the learning rates. Finally, we summarize our results and formulate our conclusions (Sec. 7). In order to keep the treatment concise, we will not report the full generating functional analysis and limit ourselves to a study of the resulting effective mean-field equations. The reader is from this moment referred to $[11,13]$ for all details about its derivation in similar instances.

\section{CMG with deterministic decision-making}

The basic ingredients of the MG are as follows. There are $N$ agents. At each round $n$ of the game all agents receive the same information pattern $\mu(n)$ drawn at random with uniform probability from a set of $P$ possible. $P$ is assumed to scale linearly with $N$, so that in the limit $N \rightarrow \infty$ the parameter $\alpha=P / N$ (the relative number of information patterns) remains finite. We assume that each agent is endowed with two different strategies (labeled by $g=1,2$ ) to convert the information into a ternary trading decision: $\boldsymbol{a}_{i g}:\{1, \ldots, P\} \ni \mu \rightarrow a_{i g}^{\mu} \in\{-1,0,1\} . a_{i g}^{\mu}$ is the action prescribed to agent $i$ by his $g$-th strategy upon receipt of information $\mu$. One might think that $a_{i g}^{\mu}= \pm 1$ correspond to 'buy' and 'sell', respectively, while $a_{i g}^{\mu}=0$ stands for 'do nothing'. For all $i, g$ and $\mu$, each $a_{i g}^{\mu}$ is selected randomly and independently from $\{-1,0,1\}$ before the start of the game and fixed. Following [3], we take a probability distribution of the form

$$
P\left(a_{i g}^{\mu}\right)=\frac{f_{i}}{2} \delta_{a_{i g}^{\mu}, 1}+\frac{f_{i}}{2} \delta_{a_{i g}^{\mu},-1}+\left(1-f_{i}\right) \delta_{a_{i g}^{\mu}, 0}
$$

The numbers $f_{i}\left(0 \leq f_{i} \leq 1\right)$ represent the trading frequencies of agents. They can be seen as an additional family of i.i.d. quenched random variables with a prescribed probability density which we denote by $q(f)$. In the original $\mathrm{MG}$, where all agents trade at all rounds, one has $q(f)=\delta(f-1)$.

The system's dynamics is defined through the microscopic stochastic equations that govern the decision making of the individual traders. Each strategy of every agent is given an initial valuation $p_{i g}(0)$, which is updated at the end of every round. Loosely speaking, $p_{i g}(n)$ measures the performance of $g$ up to round $n$. At the beginning of round $n$, every trader selects his so-far best-performing strategy, $\widetilde{g}_{i}(n)=\arg \max p_{i g}(n)$, and formulates a bid according to the trading decision it prescribes: $b_{i}(n)=a_{i \tilde{g}_{i}(n)}^{\mu(n)}$. The total bid (or, the excess demand) at round $n$ is simply $A(n)=(1 / \sqrt{N}) \sum_{i=1}^{N} b_{i}(n)$. Once $A(n)$ is known, strategy valuations are updated according to

$$
p_{i g}(n+1)=p_{i g}(n)-a_{i g}^{\mu(n)} A(n)
$$

and agents move to the next round. Strategies that would have prescribed the minority (resp. majority) action, i.e. such that $a_{i g}^{\mu(n)} A(n)<0$ (resp. $>0$ ), are thus rewarded (resp. penalized). The valuation of strategies with $a_{i g}^{\mu(n)}=$ 0 is instead left unchanged. We assume that agents neglect their market impact [5].

Introducing the 'preferences' $y_{i}(n)=\left[p_{i 1}(n)-p_{i 2}(n)\right] / 2$, and the quantities $\boldsymbol{\xi}_{i}=\left(\boldsymbol{a}_{i 1}-\boldsymbol{a}_{i 2}\right) / 2, \boldsymbol{\omega}_{i}=\left(\boldsymbol{a}_{i 1}+\boldsymbol{a}_{i 2}\right) / 2$, and $\boldsymbol{\Omega}=(1 / \sqrt{N}) \sum_{i=1}^{N} \boldsymbol{\omega}_{i}$, (2) becomes

$$
y_{i}(n+1)=y_{i}(n)-\xi_{i}^{\mu(n)}\left[\Omega^{\mu(n)}+\frac{1}{\sqrt{N}} \sum_{j=1}^{N} \xi_{j}^{\mu(n)} s_{j}(n)\right]
$$

The Ising spin $s_{i}(n)=\operatorname{sign}\left[y_{i}(n)\right] \in\{-1,1\}$ is the relevant dynamical variable: $s_{i}(n)=1$ (resp. -1 ) indicates that agent $i$ has chosen strategy $g=1$ (resp. 2) at round $n$.

The simplest dynamical approach to the analysis of the stationary states of (3) is based on the 'batch' approximation, first employed in [11]. It consists in averaging (3) over $\mu$. The result is

$$
y_{i}(t+1)=y_{i}(t)-h_{i}-\sum_{j=1}^{N} J_{i j} s_{j}(t)
$$

where $t$ is a rescaled time, $h_{i}=(2 / \sqrt{N}) \boldsymbol{\Omega} \cdot \boldsymbol{\xi}_{i}$ and $J_{i j}=$ $(2 / N) \boldsymbol{\xi}_{i} \cdot \boldsymbol{\xi}_{j}$. This choice describes strictly speaking a situation in which valuation updates are made once every $P$ steps or, equivalently, considering the average effect of all possible $\mu$ 's. It is now well known, that the stationary state of (4) is qualitatively and quantitatively very similar, though not identical, to that of (3). The analysis of (4) is however much simpler and more straightforward than that of the original 'on-line' dynamics (3).

In the limit $N \rightarrow \infty$, the Markovian multi-agent process (4) can be studied à la De Dominicis [6] by introducing the generating functional of the dynamics, i.e.

$$
\begin{aligned}
& Z[\boldsymbol{\psi}]=\left\langle e^{\mathrm{i} \sum_{i t} y_{i}(t) \psi_{i}(t)}\right\rangle_{\text {paths }}= \\
& =\int p(\boldsymbol{y}(0)) e^{\mathrm{i} \sum_{i t} \widehat{y}_{i}(t)\left[y_{i}(t+1)-y_{i}(t)-\theta_{i}(t)\right]+y_{i}(t) \psi_{i}(t)} \times \\
& \quad \times e^{\mathrm{i} \sum_{i t} \widehat{y}_{i}(t)\left[h_{i}+\sum_{j} J_{i j} s_{j}(t)\right]} \prod_{i t}\left[d y_{i}(t) d \widehat{y}_{i}(t) /(2 \pi)\right]
\end{aligned}
$$

and carrying out the average over the quenched disorder (i.e. over the $\boldsymbol{a}_{i g}$ 's with probability distribution (1)). This procedure ultimately results in a non-Markovian stochastic equation for a single effective agent whose properties are equivalent to those of the original $N$-agent system. In our case, the effective agent is trading with frequency $f$. As the calculation is standard, we will limit ourselves to the final outcome, referring the reader to e.g. [11] for details of a closely related case. The effective-agent equation turns out to be given by

$$
y(t+1)=y(t)+\sum_{t^{\prime} \leq t} R\left(t, t^{\prime}\right) s\left(t^{\prime}\right)+\theta(t)+\sqrt{\alpha f} z(t)
$$


where $R\left(t, t^{\prime}\right)$ describes the retarded self-interaction, whose precise form will be given below, $\theta(t)$ is an external field added to probe the system against small perturbations, and $z(t)$ is a zero-average Gaussian noise with correlations $\left\langle z(t) z\left(t^{\prime}\right)\right\rangle=\Lambda\left(t, t^{\prime}\right)$, with

$$
\Lambda=\left[(\mathrm{I}+\mathrm{G})^{-1}(\mathrm{~F}+\mathrm{C})\left(\mathrm{I}+\mathrm{G}^{T}\right)^{-1}\right]
$$

We used the following notation: I stands for the identity matrix, with elements $I\left(t, t^{\prime}\right)=\delta_{t t^{\prime}} ; \mathrm{F}$ denotes the matrix with elements $F\left(t, t^{\prime}\right)=\bar{f}$, with $\bar{f}$ the average trading frequency; $\mathrm{C}$ and $\mathrm{G}$ are defined by their respective components

$$
C\left(t, t^{\prime}\right)=\left\langle s(t) s\left(t^{\prime}\right)\right\rangle_{*} \quad \text { and } \quad G\left(t, t^{\prime}\right)=\left\langle\frac{\partial s(t)}{\partial \theta\left(t^{\prime}\right)}\right\rangle_{*}
$$

where the $\langle\cdots\rangle_{*}$ average is intended over the effective process (6) further averaged over $f$ :

$$
\begin{aligned}
\langle\cdots\rangle_{*} & =\int d f f q(f) \times \\
& \times \int \prod_{t} \frac{d y(t) d \widehat{y}(t)}{2 \pi} e^{-\frac{\alpha f}{2} \sum_{t t^{\prime}} \widehat{y}(t) \Lambda\left(t, t^{\prime}\right) \widehat{y}\left(t^{\prime}\right)} \times \\
& \times(\cdots) e^{\mathrm{i} \sum_{t} \widehat{y}(t)\left[y(t+1)-y(t)-\theta(t)-\sum_{t^{\prime}} R\left(t, t^{\prime}\right) s\left(t^{\prime}\right)\right]}
\end{aligned}
$$

$q(f)$ is the common probability density of the trading frequencies. As usual, $C\left(t, t^{\prime}\right)$ and $G\left(t, t^{\prime}\right)$ can be identified with the disorder-averaged correlation and response functions of the multi-agent process:

$$
\begin{aligned}
C\left(t, t^{\prime}\right) & =\frac{1}{N} \sum_{i}\left[\left\langle s_{i}(t) s_{i}\left(t^{\prime}\right)\right\rangle_{\mathrm{paths}}\right]_{\mathrm{dis}} \\
G\left(t, t^{\prime}\right) & =\frac{1}{N} \sum_{i}\left[\frac{\partial}{\partial \theta_{i}\left(t^{\prime}\right)}\left\langle s_{i}(t)\right\rangle_{\mathrm{paths}}\right]_{\mathrm{dis}}
\end{aligned}
$$

For simplicity of notation, we set

$$
\begin{aligned}
& C\left(t, t^{\prime}\right)=\int f q(f) C\left(t, t^{\prime} \mid f\right) d f \\
& G\left(t, t^{\prime}\right)=\int f q(f) G\left(t, t^{\prime} \mid f\right) d f
\end{aligned}
$$

Finally, the retarded self-interaction kernel has the form

$$
\mathrm{R}=-\alpha f(\mathrm{I}+\mathrm{G})^{-1}
$$

For $q(f)=\delta(f-1)$ the equations for the standard 'batch' MG [11] are immediately recovered.

Making for the asymptotic behavior of $\mathrm{C}$ and $\mathrm{G}$ the customary assumptions [16] of time-translation invariance,

$$
\begin{aligned}
& \lim _{t \rightarrow \infty} C(t+\tau, t)=C(\tau) \\
& \lim _{t \rightarrow \infty} G(t+\tau, t)=G(\tau)
\end{aligned}
$$

finite integrated response,

$$
\lim _{t \rightarrow \infty} \sum_{t^{\prime} \leq t} G\left(t, t^{\prime}\right)<\infty
$$

and weak long-term memory

$$
\lim _{t \rightarrow \infty} G\left(t, t^{\prime}\right)=0 \quad \forall t^{\prime} \text { finite }
$$

ergodic stationary states of the dynamics can be fully characterized in terms of a few parameters. These are, in particular, the persistent autocorrelation

$$
c=\lim _{\tau \rightarrow \infty} \frac{1}{\tau} \sum_{t<\tau} C(t)=\int f q(f) c(f) d f
$$

with $c(f)=\lim _{\tau \rightarrow \infty}(1 / \tau) \sum_{t<\tau} C(t \mid f)$ the autocorrelation of the effective trader (trading with frequency $f$ ), and the susceptibility (or integrated response)

$$
\chi=\lim _{\tau \rightarrow \infty} \sum_{t \leq \tau} G(t)=\int f q(f) \chi(f) d f
$$

where $\chi(f)=\lim _{\tau \rightarrow \infty} \sum_{t \leq \tau} G(t \mid f)$ is the effective trader's susceptibility.

Following [11], one can easily derive from (6) an equation for the long-time behavior of the rescaled variable $\widetilde{y}(t)=y(t) / t$ :

$$
\frac{\widetilde{y}}{\sqrt{\alpha f}}=-\gamma s+z, \quad \gamma=\frac{\sqrt{\alpha f}}{1+\chi}
$$

where $\widetilde{y}=\lim _{t \rightarrow \infty} \widetilde{y}(t)$, while $s$ and $z$ denote time-averages of $s(t)$ and $z(t)$, respectively. As in the standard MG, part of the agents will 'freeze' at $s= \pm 1$ and will end up using just one of their strategies (for these, $|y(t)| \rightarrow \infty$ asymptotically in such a way that $\widetilde{y}$ remains finite), whereas the remaining agents will keep flipping between their strategies (for these, $y(t)$ remains asymptotically finite and $\widetilde{y}$ is zero). It is easy to see that agents are 'frozen' for $|z|>\gamma$ while they are 'fickle' (with $s=z / \gamma$ ) for $|z|<\gamma$. The asymptotic values of the interesting quantities can hence be immediately derived by separating the contributions of frozen and fickle agents. For example, denoting by \langle\rangle an average over the Gaussian r.v. $z$ with zero mean and variance (from $(7))\left\langle z^{2}\right\rangle=(\bar{f}+c) /(1+\chi)^{2}$ and defining

$$
\phi(f)=\langle\theta(|z|-\gamma)\rangle=1-\operatorname{erf} \frac{\gamma}{\sqrt{2\left\langle z^{2}\right\rangle}}
$$

i.e. the fraction of agents trading with frequency $f$ that are frozen (strictly speaking, the probability that the effective agent is frozen), one has

$$
\begin{aligned}
c(f) \equiv\left\langle s^{2}\right\rangle & =\langle\theta(|z|-\gamma)\rangle+\left\langle(z / \gamma)^{2} \theta(\gamma-|z|)\right\rangle= \\
& =\phi(f)+\frac{\bar{\phi}(f)}{\lambda(f)^{2}}-\frac{1}{\lambda(f)} \sqrt{\frac{2}{\pi}} e^{-\lambda(f)^{2} / 2}
\end{aligned}
$$

with $\bar{\phi}(f)=1-\phi(f)=\langle\theta(\gamma-|z|)\rangle$ and

$$
\lambda(f)=\frac{\gamma}{\sqrt{\left\langle z^{2}\right\rangle}}=\sqrt{\frac{\alpha f}{\bar{f}+c}}
$$


Analogously, $\chi(f)$ can be calculated from the formula

$$
\gamma \sqrt{\alpha f} \chi(f)=\langle\theta(\gamma-|z|)\rangle=\bar{\phi}(f)
$$

which is derived by noticing that the external field and the noise term enter (6) in the same way, apart from the $\sqrt{\alpha f}$ factor. The calculation of $\partial s / \partial z$ is then trivial and leads to (25), which in turn gives

$$
\chi(f)=\frac{1+\chi}{\alpha f} \operatorname{erf} \frac{\lambda(f)}{\sqrt{2}}
$$

Inserting (23) and (26) into (19) and (20) one gets two equations for $c$ and $\chi$ which must be solved selfconsistently. Solutions will clearly depend on $\alpha$ and on the underlying frequency distribution $q(f)$. It is the purpose of the next two sections to study these equations and the corresponding solutions in two interesting cases.

\section{Bimodal frequency distribution}

As a start, let us consider the simplest case, where

$$
q(f)=(1-q) \delta(f-1)+q \delta\left(f-f_{0}\right)
$$

with $0 \leq q \leq 1$ and $0 \leq f_{0} \leq 1$, describing a mixed population formed by frequent traders, who buy or sell at every round, and occasional traders, who instead have a finite probability $1-f_{0}$ of taking no action. The population fractions of the two types are $(1-q)$ and $q$, respectively.

The total fraction of frozen agents is evidently

$$
\begin{aligned}
\phi=(1 & -q) \phi(1)+q \phi\left(f_{0}\right)= \\
& =1-\operatorname{erf} \frac{\lambda(1)}{\sqrt{2}}+q\left[\operatorname{erf} \frac{\lambda(1)}{\sqrt{2}}-\operatorname{erf} \frac{\lambda\left(f_{0}\right)}{\sqrt{2}}\right]
\end{aligned}
$$

where $\lambda(f)$ is given by $(24)$ with $\bar{f}=1-q\left(1-f_{0}\right)$. Eq. (19) is given by

$$
c=(1-q) c(1)+q f_{0} c\left(f_{0}\right)
$$

with $c(f)$ given by (23), while, finally, the susceptibility turns out to be expressed as

$$
\chi=(1-q) \chi(1)+q f_{0} \chi\left(f_{0}\right)
$$

with $\chi(f)$ given by $(26)$.

Equations (29) and (30) must be solved self-consistently and are valid as long as none of the assumptions (15-18) is violated. In the MG, ergodicity breaking is related to the onset of anomalous response, i.e. to a divergence of $\chi$. Working out (30) explicitly, one finds the relation

$$
\frac{\alpha \chi}{1+\chi}=(1-q) \operatorname{erf} \frac{\lambda(1)}{\sqrt{2}}+q \operatorname{erf} \frac{\lambda\left(f_{0}\right)}{\sqrt{2}}
$$

$\chi$ is evidently finite for large $\alpha$. The critical values of the control parameter where $\chi$ diverges are immediately obtained as

$$
\alpha_{c}\left(q, f_{0}\right)=(1-q) \operatorname{erf}(x)+q \operatorname{erf}\left(x \sqrt{f_{0}}\right)
$$

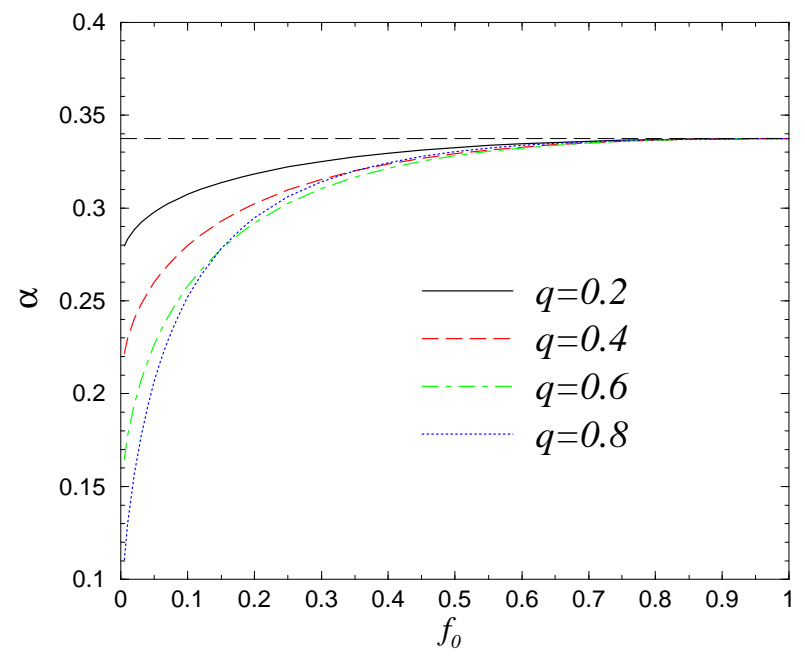

Fig. 1. Phase diagram of the multi-frequency MG with bimodal frequency distribution. The curves $\alpha_{c}$ vs $f_{0}$ (trading frequency of occasional traders) are shown for different values of the fraction $q$ of occasional traders. For $\alpha>\alpha_{c}$ the system is ergodic. The horizontal dashed line at $\alpha \simeq 0.3374$ marks the position of the critical point of the standard MG and coincides with the critical line in the case $q=0$.

where $x$ solves (29) at $\alpha_{c}$. After some algebra, the latter condition is shown to be, in terms of $x$, equivalent to the following:

$$
\begin{aligned}
2 \bar{f}-(1-q) \operatorname{erf}(x)-q f_{0} \operatorname{erf}\left(x \sqrt{f_{0}}\right)+ \\
\\
\quad-\frac{1-q}{x \sqrt{\pi}} e^{-x^{2}}-\frac{q}{x} \sqrt{\frac{f_{0}}{\pi}} e^{-f_{0} x^{2}}=0
\end{aligned}
$$

For $\alpha>\alpha_{c}$, the system is ergodic and admits a unique stationary state. At $\alpha_{c}$, the integrated response diverges and anomalous response sets in. For $\alpha<\alpha_{c}$, finally, ergodicity is broken and the long-time limit of macroscopic quantities depends on the initial conditions of the dynamics. Clearly, the limiting cases $q=0$ and $f_{0}=1$ reproduce, as they should, the results of the standard MG, while the limit $q=1$ brings us back to the standard case after a trivial re-scaling. Notice that, as usual in MGs, $\chi$ diverges when $\alpha$ equals the fraction $1-\phi$ of fickle traders.

Numerical solution of (33) and subsequent insertion of the result into (32) leads to the $\left(f_{0}, \alpha\right)$ phase diagrams shown in Fig. 1 for different values of $q$. For sufficiently small $f_{0}$, the critical point $\alpha_{c}$ decreases as $q$ increases, signaling that the ergodic phase gets larger while the efficient phase shrinks. As $f_{0}$ grows, $\alpha_{c}$ tends instead to the standard MG value $0.3374 \ldots$ for any $q$, as was easily predictable.

Solving instead (28), (29), and (30) one obtains the behavior of the macroscopic order parameters and of the fraction of frozen agents as a function of $\alpha$ upon varying $q$ and $f_{0}$. These quantities give an idea of the interplay between the two types of agents. For sakes of conciseness and because we believe it is the most interesting instance, we concentrate on the case where the trading frequency 


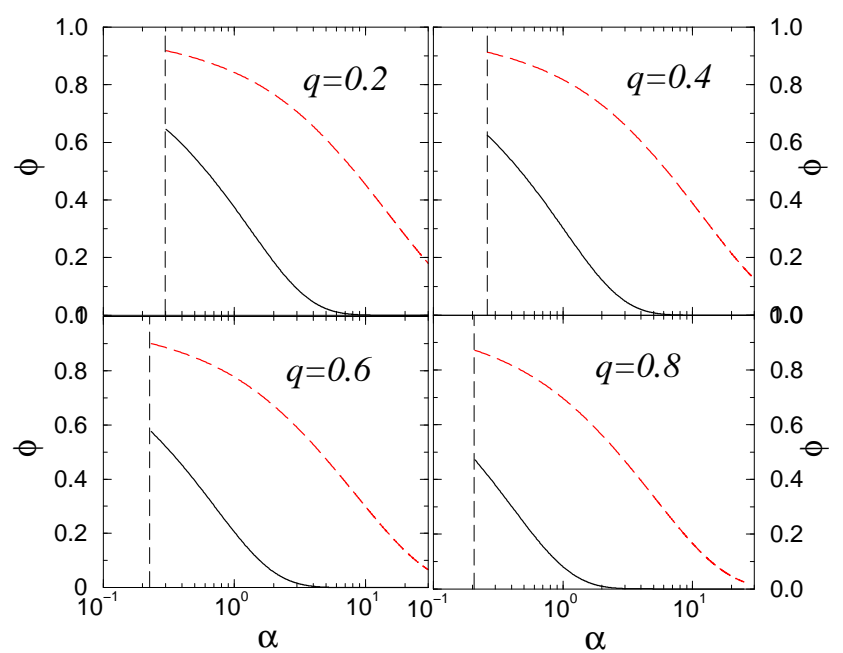

Fig. 2. Fraction $\phi(f)$ of agents with trading frequency $f$ that are frozen for values of $q$ as reported in the figures. The vertical dashed lines give the positions of the critical points, below which (17) is violated and the theory is no longer valid. The continuous (resp. dashed) line represents $\phi(1)$ (resp. $\phi(0.05))$.

of occasional traders is much lower than that of regulars, that is we assume regulars and occasional traders operate on widely different time scales. We take in particular $f_{0}=$ 0.05. Results for $\phi(f)$ are shown in Fig. 2. It is clear that occasional traders are more likely to freeze than regular traders, as also argued in the static solution outlined in [3]. This tendency is robust with respect to changes of $q$ and $f_{0}$. It is interesting to notice that a larger fraction of occasional traders freeze when regulars outnumber them.

In order to test our predictions, in particular for what concerns the phase transition, and to understand further the combined effects of regulars and irregulars on global efficiency in this simple case, we can study the volatility matrix, defined as [11]

$$
\equiv=\frac{1}{2} \wedge=\frac{1}{2}\left[(I+G)^{-1}(F+C)\left(I+G^{T}\right)^{-1}\right]
$$

三 has two important properties. First, the magnitude of global fluctuations, i.e. $\sigma^{2}=\left\langle A^{2}\right\rangle_{\text {time }}$, which serves as a measure of global efficiency (the smaller is $\sigma^{2}$, the more efficient is the allocation of resources, that is, the smaller the amount of resource that is wasted), is given by

$$
\sigma^{2}=\lim _{t \rightarrow \infty} \Xi(t, t)
$$

Second, the quantity $H=(1 / P) \sum_{\mu=1, P}\langle A \mid \mu\rangle_{\text {time }}^{2}$, where

$$
\langle A \mid \nu\rangle_{\text {time }}=\lim _{L \rightarrow \infty} \frac{1}{L-L_{\mathrm{eq}}} \sum_{n=L_{\mathrm{eq}}, L} A(n) \delta_{\mu(n), \nu}
$$

that quantifies the informational efficiency of the system (when $H=0$ the minority action is not predictable on the basis of the state of the world $\mu(n)$ alone and the system

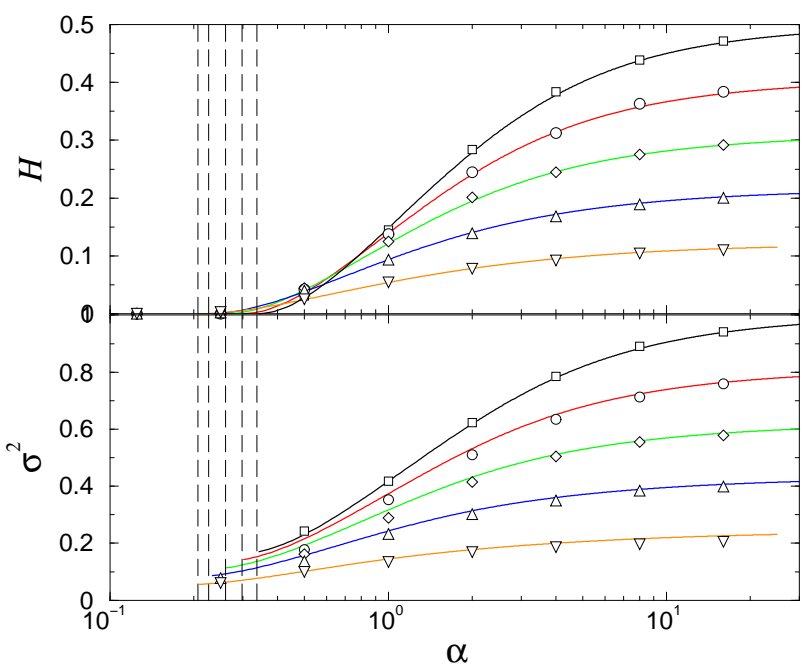

Fig. 3. Behavior of $H$ and $\sigma^{2}$ vs $\alpha$ at fixed $f_{0}=0.05$ for $q=0,0.2,0.4,0.6,0.8$ (continuous curves, top to bottom). The vertical dashed lines mark the positions of the critical points. In the non-ergodic regime $\alpha<\alpha_{c}$ the theory is no longer valid. Markers denote the results of computer simulations of a system of 256 agents for the same values of $f_{0}$ and $q$, averaged over 100 disorder samples.

is efficient, in the sense that it does not create information an external agent could exploit to have a gain) is roughly given by the persistent part of $\Xi$. Both these properties can be proved under general conditions, as done for instance in [11].

$H$ is easily seen from (34) to be given by

$$
H=\frac{\bar{f}+c}{2(1+\chi)^{2}}
$$

and tends to $\bar{f} / 2$ for $\alpha \rightarrow \infty$. $\sigma^{2}$ can instead be approximated with

$$
\sigma^{2}=\frac{\bar{f}+\int f q(f) \phi(f) d f}{2(1+\chi)^{2}}+\frac{1}{2}\left[\bar{f}-\int f q(f) \phi(f) d f\right]
$$

and tends to $\bar{f}$ for $\alpha \rightarrow \infty$. The proof of (38), which requires an approximate evaluation of (35), is a slight modification of the standard case, reported in [11], and we will not report it here. For the present model, we have obtained $\sigma^{2}$ and $H$ from numerical simulations (performed with flat or unbiased initial conditions $y_{i}(0)=0$ for all $i$ ). The comparison with analytical predictions can be seen in Fig. 3. As in the standard MG, the ergodicity breaking transition coincides with a transition between an informationally efficient phase with $H=0\left(\alpha<\alpha_{c}\right)$ and an informationally inefficient one with $H>0\left(\alpha>\alpha_{c}\right)$. $H$ vanishes at at $\alpha_{c}$, where $\chi \rightarrow \infty$, and decreases as $q$ increases. At the same time, $\sigma^{2}$ decreases in vicinity of the critical point, signaling an increased degree of cooperation. 


\section{Power-law frequency distribution}

Let us now turn our attention to the case where

$$
q(f)=\kappa f^{\kappa-1}, \quad \bar{f}=\frac{\kappa}{\kappa+1}
$$

where the frequency distribution is scale-free (i.e., there is no single characteristic time scale). The standard instance is recovered in the limit $\kappa \rightarrow \infty$, in which $q(f) \rightarrow \delta(f-1)$. This problem was treated statically in [3], so we will limit ourselves to calculating the phase diagram and discussing its qualitative properties. The general dynamical solution is now slightly more complicated than the bimodal case, however it is still possible to write down closed equations for the relevant parameters. The behavior of macroscopic observables turns however out to be similar to the bimodal case. Let us first notice that, since

$$
1-\phi=\kappa \int_{0}^{1} f^{\kappa-1} \operatorname{erf} \frac{\lambda(f)}{\sqrt{2}} d f
$$

and since, from (26),

$$
\frac{\alpha \chi}{1+\chi}=1-\phi
$$

we immediately obtain the expression for the critical line signaling ergodicity breaking in the $(\kappa, \alpha)$ space, namely

$$
\alpha_{c}(\kappa)=\kappa \int_{0}^{1} f^{\kappa-1} \operatorname{erf}(x \sqrt{f}) d f
$$

where again $x$ solves the equation for $c$, namely

$$
c=\kappa \int f^{\kappa} c(f) d f
$$

at $\alpha_{c}$. One can see by a short calculation that in terms of $x$ and for $\alpha=\alpha_{c}$, Eq. (42), equation (43) reads

$$
\frac{2 \kappa}{1+\kappa}-\kappa \int_{0}^{1} f^{\kappa-1}\left[f \operatorname{erf}(x \sqrt{f})+\frac{\sqrt{f}}{x \sqrt{\pi}} e^{-f x^{2}}\right] d f=0
$$

When $\kappa \rightarrow \infty$, the standard picture is recovered. Using a more compact notation, (44) be re-cast as

$$
\frac{\kappa}{1+\kappa}[2-\operatorname{erf}(x)]+\frac{\mathcal{F}(\kappa, x ; 3 / 2)}{1+\kappa}-\mathcal{F}(\kappa, x ; 1 / 2)=0
$$

where we introduced the shorthand

$$
\mathcal{F}(\kappa, x ; a)=\frac{\kappa}{x^{2(\kappa+1)} \sqrt{\pi}}\left[\Gamma(\kappa+a)-\Gamma^{u}\left(\kappa+a, x^{2}\right)\right]
$$

with $\Gamma(\cdot)$ and $\Gamma^{u}(\cdot, \cdot)$ the Euler Gamma function and the upper incomplete Gamma function, respectively. (45) can be solved numerically for $x$ upon varying $\kappa$. Subsequently, the corresponding value of $\alpha_{c}$ can be calculated from (42):

$$
\alpha_{c}(\kappa)=\operatorname{erf}(x)-\frac{x^{2}}{\kappa} \mathcal{F}(\kappa, x ; 1 / 2)
$$

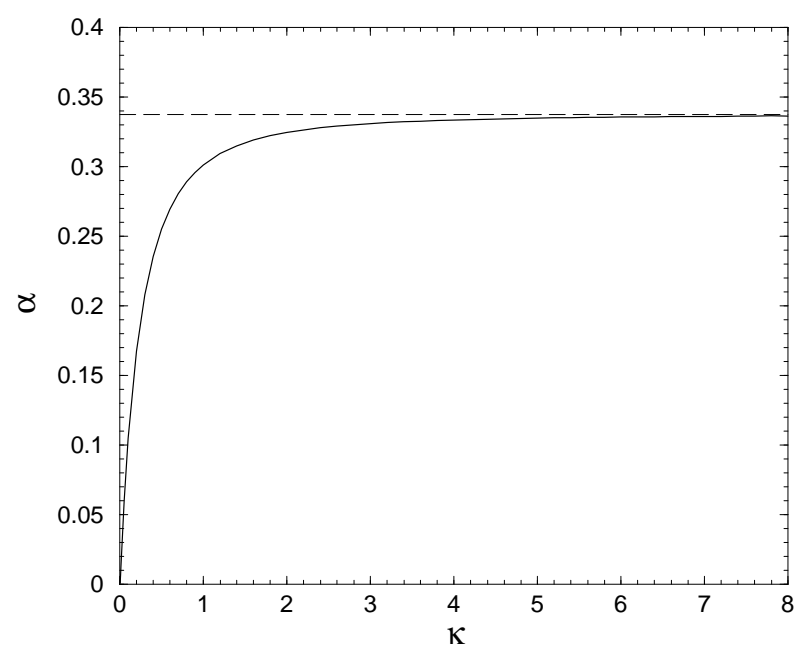

Fig. 4. Phase diagram of the multi-frequency MG with powerlaw frequency distribution. The curve $\alpha_{c}$ vs $\kappa$ is shown. For $\alpha>\alpha_{c}$ the system is ergodic and informationally inefficient. The horizontal dashed line at $\alpha \simeq 0.3374$ marks the position of the critical point of the standard MG.

The resulting phase diagram is shown in Fig. 4. For large $\kappa$, the standard MG picture is recovered, as expected. As $\kappa$ decreases, $\alpha_{c}$ also decreases, proving that the fraction of active agents is also getting smaller and smaller. $\alpha_{c}$ finally tends to zero when $\kappa \rightarrow 0$. While this picture is qualitatively identical to that derived in [3], there are certain numerical differences as in our solution $\alpha_{c}(\kappa)$ seems to approach the standard limit $\alpha_{c}(\infty) \simeq 0.3374$ slightly faster than in the replica solution. However a direct comparison could be misleading since the order parameters are defined differently in the two cases. This point might deserve further investigation. The fraction $\phi$ of frozen agents behaves similarly to what shown in Fig. 2, in that agents trading less frequently are more likely to be frozen, as also discussed in [3]. Clearly, the formulas derived in the previous section for $\sigma^{2}$ and $H$ can be applied, mutatis mutandis, also in this case.

\section{CMG with finite learning rates}

We now add one layer of difficulty by introducing a learning rate in the individual agents' dynamics of strategy selection, as first done for the standard model in [17]. Up to now, agents have strictly followed their best performing strategy at each round, as encoded in the deterministic rule

$$
s_{i}(n)=\operatorname{sign}\left[y_{i}(n)\right]
$$

Now we account for the possibility that at each time step the value of $s_{i}(n)$ is established by a probabilistic rule. 
The simplest choice for the latter is ${ }^{1}$

$$
\operatorname{Prob}\left\{s_{i}(n)= \pm 1\right\} \sim e^{ \pm \Gamma_{i} y_{i}(n)}
$$

which turns (48) into

$$
s_{i}\left(n \mid \zeta_{i}(n), \Gamma_{i}\right)=\operatorname{sign}\left[y_{i}(n)+\zeta_{i}(n) / \Gamma_{i}\right]
$$

where the $\zeta_{i}$ 's are i.i.d. Gaussian r.v.'s with probability density $p(\zeta)=\left[1-\tanh ^{2}(\zeta)\right] / 2$ (zero average, unit variance). The positive constants $\Gamma_{i}$ can be interpreted as 'learning rates', upon varying which one interpolates between the deterministic rule (48), that is recovered for $\Gamma_{i} \rightarrow \infty$, and a fully randomized rule, corresponding to $\Gamma_{i}=0$. The dynamics of the standard model with finite learning rates was tackled in [13], where the phrase 'additive decision noise' was introduced to describe the situation of (50). Notice that this type of noise affects fickle traders only, because for 'frozen' traders $\left|y_{i}(n)\right| \rightarrow \infty$, hence it is to be expected that the critical points obtained for different trading frequencies do not change. A more complicated situation is that of 'multiplicative decision noise', first introduced in [18], where instead of (50) one has

$$
s_{i}\left(n \mid \zeta_{i}(n), \Gamma_{i}\right)=\operatorname{sign}\left[y_{i}(n)\left(1+\zeta_{i}(n) / \Gamma_{i}\right)\right]
$$

which corresponds to

$$
\operatorname{Prob}\left\{s_{i}(n)= \pm 1\right\} \sim e^{ \pm \Gamma_{i} \operatorname{sign}\left[y_{i}(n)\right]}
$$

This choice clearly affects also frozen agents.

The dynamical theory of this case is a straightforward modification of that constructed for the deterministic case. In general, with $s\left(n \mid \zeta_{i}(n), \Gamma_{i}\right)$, the dynamics (4) becomes

$$
y_{i}(t+1)=y_{i}(t)-h_{i}-\sum_{j=1}^{N} J_{i j} s_{j}\left(t \mid \zeta_{j}(t), \Gamma_{j}\right)
$$

Again, one can write down its generating functional, taking into account the fact that this time the calculation of one-step transition probabilities, the product of which is used to compute averages over paths, requires carrying out an average over the $\zeta_{i}$ 's (we denote this operation by $\left.[\cdots]_{\zeta}\right)$ :

$$
\begin{aligned}
& Z[\boldsymbol{\psi}]=\left\langle e^{\mathrm{i} \sum_{i t} y_{i}(t) \psi_{i}(t)}\right\rangle_{\text {paths }}= \\
& =\int p(\boldsymbol{y}(0)) e^{\mathrm{i} \sum_{i t} \widehat{y}_{i}(t)\left[y_{i}(t+1)-y_{i}(t)+h_{i}-\theta_{i}(t)\right]+y_{i}(t) \psi_{i}(t)} \times \\
& \times\left[e^{\mathrm{i} \sum_{i t} \widehat{y}_{i}(t) \sum_{j} J_{i j} s_{j}\left(t \mid \zeta_{j}(t), \Gamma_{j}\right)}\right]_{\zeta} \prod_{i t}\left[d y_{i}(t) d \widehat{y}_{i}(t) /(2 \pi)\right]
\end{aligned}
$$

Again, averaging over the quenched disorder leads to a non-Markovian process that now describes a single effective agent trading with frequency $f$ and learning at rate $\Gamma$.

\footnotetext{
1 We use for the learning rate the customary symbol $\Gamma$. The reader is warned that it is different from the $\Gamma$ function appearing in Sec. 4.
}

And again, we skip all formalities of the derivation of this process and focus on its long-time properties. The analog of (6) in this case is

$y(t+1)=y(t)+\sum_{t^{\prime} \leq t} R\left(t, t^{\prime}\right) s\left(t^{\prime} \mid \zeta\left(t^{\prime}\right), \Gamma\right)+\theta(t)+\sqrt{\alpha f} z(t)$

(7) and (14) are still formally valid, but the correlation and response functions $C\left(t, t^{\prime}\right)$ and $G\left(t, t^{\prime}\right)$ are defined respectively as

$$
\begin{gathered}
C\left(t, t^{\prime}\right)=\left\langle s(t \mid \zeta(t), \Gamma) s\left(t^{\prime} \mid \zeta\left(t^{\prime}\right), \Gamma\right)\right\rangle_{*} \\
G\left(t, t^{\prime}\right)=\frac{\partial}{\partial \theta\left(t^{\prime}\right)}\langle s(t \mid \zeta(t), \Gamma)\rangle_{*}
\end{gathered}
$$

where the average $\langle\cdots\rangle_{*}$, formerly given by (9), involves now also averages over $\boldsymbol{z}$ and $\Gamma$. In particular, if we denote by $w(\Gamma)$ the probability density of $\Gamma$, we have

$$
\begin{gathered}
\langle\cdots\rangle_{*}=\int_{0}^{1} d f f q(f) \int_{0}^{\infty} d \Gamma w(\Gamma) \int \prod_{t} \frac{d y(t) d \widehat{y}(t)}{2 \pi} \times \\
\times\left[(\cdots) e^{-\frac{\alpha f}{2} \sum_{t t^{\prime}} \widehat{y}(t) \Lambda\left(t, t^{\prime}\right) \widehat{y}\left(t^{\prime}\right)} \times\right. \\
\left.\times e^{\mathrm{i} \sum_{t} \widehat{y}(t)\left[y(t+1)-y(t)-\theta(t)-\sum_{t^{\prime}} R\left(t, t^{\prime}\right) s\left(t^{\prime} \mid \zeta\left(t^{\prime}\right), \Gamma\right)\right]}\right]_{\zeta}
\end{gathered}
$$

As before, $C\left(t, t^{\prime}\right)$ and $G\left(t, t^{\prime}\right)$ represent the disorder-averaged correlation and response functions of the original system.

Proceeding as done in Sec. 2 by assuming the validity of $(15-18)$, one can derive an equation for the quantity $\widetilde{y}=\lim _{t \rightarrow \infty} y(t) / t$, which reads

$$
\frac{\widetilde{y}}{\sqrt{\alpha f}}=-\gamma m(\Gamma)+z
$$

where all quantities have the same meaning as in (21) except that now $m(\Gamma)$ is the time-average of $s(t \mid \zeta(t), \Gamma)$. This change presents no additional difficulty. In the next section we will analyze the stationary states and the phase diagrams resulting from (50) and (51) separately.

\section{Additive and multiplicative decision noises}

In the case of (50), one immediately sees that the conditions for an agent to be frozen or fickle are identical to those given in Sec. 2, hence the picture emerging from different trading frequencies (in particular the expressions for $\chi, c$ and $\phi$ ) is left unchanged since $\Gamma$ can be integrated out. In particular, the phase diagrams presented in Sec. 3 and 4 for bimodal and power-law frequency distribution remain valid. Hence, as in the standard batch $\mathrm{MG}$, the introduction of additive noise does not affect the macroscopic properties of the multi-frequency model in the ergodic regime. (On the other hand, the introduction of $\Gamma$ does affect the stationary states of the standard model in the non-ergodic regime, as first shown by computer simulations in [17] and by analytical arguments in [19].) 
Finally, we turn to the case of multiplicative decision noise (51). Following [13], it is convenient to switch to the inverse learning rate $T=1 / \Gamma$ and to introduce the quantity

$$
h(T)=\int \operatorname{sign}(1+T \zeta) p(\zeta) d \zeta
$$

which has the obvious properties $h(T) \in[0,1]$ with $h(0)=$ 1 and $h(\infty)=0$. Using this, one concludes from (59) with $m(\Gamma) \equiv m(T)=\operatorname{sign}[\widetilde{y} h(T)$ that frozen agents with $\operatorname{sign}[\widetilde{y}]= \pm 1$ have $m(T)= \pm h(T)$, respectively, and occur for $|z|>\gamma h(T)$, whereas fickle agents have $m(T)=z / \gamma$ and occur for $|z|<\gamma h(T)$.

In order to make contact with the quantities calculated in the deterministic decision-making case, we compute the autocorrelation of the effective trader with trading frequency $f, c(f)$, which can as usual be obtained by separating the contributions of frozen and fickle agents, this time performing an additional average over $T$. For simplicity, one can pass from the variable $T$ with probability density $\widetilde{w}(T)$ to $h$, Eq. (60), with probability density

$$
\rho(h)=\int_{0}^{\infty} \delta\left[h-\int \operatorname{sign}(1+T \zeta) p(\zeta) d \zeta\right] \widetilde{w}(T) d T
$$

so that

$$
c(f)=\int\left[\left\langle\theta(|z|-\gamma h) h^{2}\right\rangle+\left\langle\theta(\gamma h-|z|)(z / \gamma)^{2}\right\rangle\right] \rho(h) d h
$$

where $\langle\cdots\rangle$ denotes an average over the Gaussian r.v. $z$ with zero average and variance $\left\langle z^{2}\right\rangle=(\bar{f}+c) /(1+\chi)^{2}$, where

$$
c=\int f q(f) c(f) d f
$$

is the global persistent autocorrelation. The fraction of frozen agents reads

$$
\phi=\int d f q(f) \int d h \rho(h)\langle\theta(|z|-\gamma h)\rangle
$$

where it should be kept in mind that $\gamma$ depends on $f$ $(\gamma=\sqrt{\alpha f} /(1+\chi))$.

An expression for the total susceptibility $\chi$ can be found starting from (25) and averaging properly over $f$ and $h$ :

$$
\frac{\alpha \chi}{1+\chi}=1-\int d f q(f) \int d h \rho(h)\langle\theta(|z|-\gamma h)\rangle \equiv 1-\phi
$$

so that as usual $\chi$ diverges when the fraction of fickle agents equals $\alpha$. We have thus come to the following expression for the critical line:

$$
\alpha_{c}[q(f), w(\Gamma)]=\int d f q(f) \int d h \rho(h) \operatorname{erf}(x h \sqrt{f})
$$

where we have emphasized that $\alpha_{c}$ now depends on the trading frequency distribution $q(f)$ and on the distribution of learning rates $w(\Gamma)$ (via $\widetilde{w}(T)$ and $\rho(h)$ ). Again, the value of $x$ must be obtained by solving (63) at $\alpha_{c}$.
For the sake of brevity, we specialize the above theory only to the simplest cases where both $f$ and $T$ have binomial distributions:

$$
\begin{gathered}
q(f)=(1-q) \delta(f-1)+q \delta\left(f-f_{0}\right) \\
\widetilde{w}(T)=(1-\epsilon) \delta(T)+\epsilon \delta\left(T-T_{0}\right)
\end{gathered}
$$

with $q, f_{0}$ and $\epsilon$ real numbers belonging to the interval $[0,1]$. This describes a mixed population of frequent and occasional (trading frequency $f_{0}$ ) traders, with either deterministic decision making or stochastic decision making with learning rate $\Gamma_{0}=1 / T_{0}$. Notice that trading frequency and learning rate are treated as independent variables (this seems to the author a rather strong and possibly unrealistic assumption). Under these hypotheses, one has

$$
\rho(h)=(1-\epsilon) \delta(h-1)+\epsilon \delta\left(h-h_{0}\right), \quad h_{0}=h\left(T_{0}\right)
$$

Introducing again $\lambda(f)=\sqrt{\frac{\alpha f}{\bar{f}+c}}$ and setting

$$
\begin{aligned}
c(f, h) & =h^{2}\left[1-\operatorname{erf} \frac{\lambda(f) h}{\sqrt{2}}\right]+ \\
& +\frac{1}{\lambda(f)^{2}} \operatorname{erf} \frac{\lambda(f) h}{\sqrt{2}}-\frac{h}{\lambda(f)} \sqrt{\frac{2}{\pi}} e^{-\lambda(f)^{2} h^{2} / 2}
\end{aligned}
$$

one finds, for $c$,

$$
\begin{aligned}
& c=(1-q)(1-\epsilon) c(1,1)+(1-q) \epsilon c\left(1, h_{0}\right)+ \\
& +f_{0} q(1-\epsilon) c\left(f_{0}, 1\right)+f_{0} q \epsilon c\left(f_{0}, h_{0}\right)
\end{aligned}
$$

and for $\alpha_{c}$, which now depends on the population fractions $q$ and $\epsilon$ and on $f_{0}$ and $h_{0}$,

$$
\begin{array}{r}
\alpha_{c}=(1-q)(1-\epsilon) \operatorname{erf}(x)+(1-q) \epsilon \operatorname{erf}\left(x h_{0}\right)+ \\
+q(1-\epsilon) \operatorname{erf}\left(x \sqrt{f_{0}}\right)+q \epsilon \operatorname{erf}\left(x h_{0} \sqrt{f_{0}}\right)
\end{array}
$$

When $\alpha=\alpha_{c}$, (71) takes a simple but rather lengthy expression which we do not report. Ultimately, all equations can be solved numerically. As already done in Sec. 3, we show the phase diagram for the case where regular and occasional traders operate on very different time scales and again choose $f_{0}=0.05$ (see Fig. 5). One can clearly see that the phase structure of the MG with multiplicative noise [13] is preserved, the main effect being a shift of the position of the critical lines in the $\left(\alpha, \Gamma_{0}\right)$ space to lower values of $\alpha$ (signaling again a reduction of the informationally efficient phase).

\section{Summary and discussion}

In summary, the MG with agents trading at different frequencies and learning at different rates has been analyzed using generating-functional techniques. The dynamical critical behavior of the model is not altered by the introduction of multiple time scales for trading, but the specific phase diagram turns out to depend on the details 


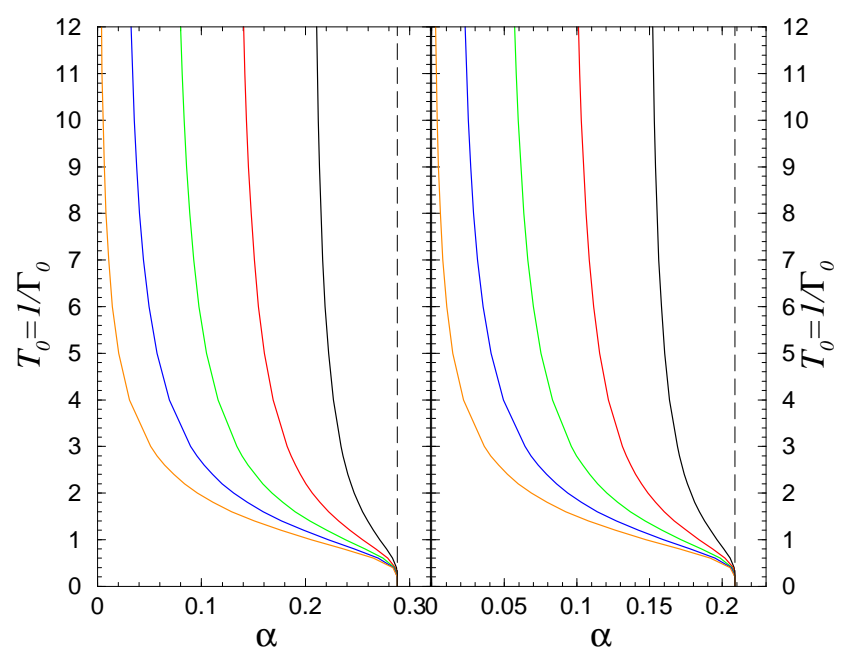

Fig. 5. Phase diagram of the multi-frequency $M G$ with stochastic decision-making, with bimodal distributions for frequencies and learning rates, with parameter $f_{0}=0.05$. Results are shown for $q=0.25$ (left panel) and $q=0.75$ (right panel). In each panel, continuous curves denote the critical lines for multiplicative decision noise for $\epsilon=0.2,0.4,0.6,0.8,1$ (right to left). The dashed vertical line marks the position of the critical point for the CMG with deterministic decision-making, which coincides with the critical line for the corresponding CMG with additive decision noise.

of the underlying frequency and learning rate distribution. We have derived the phase structure for a system of agents with deterministic decision-making when the frequency distribution is a simple bimodal and when it has a power-law form. For the case of stochastic decisionmaking, i.e. finite learning rates, the phase diagram has been computed explicitly for a double bimodal distribution of frequencies and learning rates, describing fast and slow traders with a heterogeneous distribution of stochasticity in their learning process. In accordance with [3], we found that occasional traders display a stronger tendency to freeze, namely to use just one of their strategies, than regular traders, thus contributing and additional signal to unfrozen players. To the latter, thus mostly to regular traders, is ultimately ascribable the building up of fluctuations (via freezing) and thus the decrease of global efficiency.

On a purely technical level, it is clear that combining static and dynamical methods of statistical mechanics of disordered systems leads to a very satisfactory understanding of the the macroscopic properties of MG-based market models. On the other hand, MGs are simple enough to hope they can serve as the elementary building blocks of more complicated and realistic agent-based market models, with different types of agents that are heterogeneous in strategies, beliefs, learning rates, market-impact evaluation, etc., and where the plethora of macroscopic phenomena observed in real systems could be analyzed and traced back to the individual laws of motion. Present works concerning the macroscopic properties of different versions of the MG should be seen as the necessary preliminary work to develop a full understanding at later stages.

Indeed, several directions are open for further research. For example, it would be interesting to relate $\Gamma$ and $f$ by letting agents with a smaller learning rate trade more (or less) frequently. This could also be done by assuming that $\Gamma$ and $f$ are not independent qualities, as done in Sec. 6 of the present work, but come with a joint probability density. Another worthy extension could involve the introduction of (using the terminology of [20]) 'producers', i.e. agents who have just one strategy at their disposal. It is likely that they could again affect the phase structure.

Acknowledgments. I am grateful to A. Cavagna, A.C.C. Coolen, I. Giardina, J.A.F. Heimel, M. Marsili and G. Mosetti for encouragement and many useful discussions and precious suggestions.

\section{References}

1. D. Challet and Y.-C. Zhang, Physica A 246407 (1997)

2. See http://www.unifr.ch/econophysics/minority/ for an extensive and commented list of references.

3. M. Marsili and M. Piai, Physica A 310234 (2002)

4. D. Challet, M. Marsili and R. Zecchina, Phys. Rev. Lett. 841824 (2000)

5. M. Marsili, D. Challet and R. Zecchina, Physica A 280 $522(2000)$

6. C. De Dominicis, Phys. Rev. B 184913 (1978)

7. T.R. Kirkpatrick and D. Thirumalai, Phys. Rev. B 365388 (1987)

8. A. Crisanti, H. Horner and H.-J. Sommers, Z. Phys B 92 257 (1993)

9. L. Cugliandolo and J. Kurchan, Phys. Rev. Lett. 71173 (1993)

10. J.-P. Bouchaud, L. Cugliandolo, J. Kurchan and M. Mezard, in A.P. Young (Ed.), Spin glasses and random fields, World Scientific (Singapore), 1998.

11. J.A.F. Heimel and A.C.C. Coolen, Phys. Rev. E 63056121 (2001)

12. J.A.F. Heimel and A. De Martino, J. Phys. A 34 L539 (2001)

13. A.C.C. Coolen, J.A.F. Heimel and D. Sherrington, Phys. Rev. E 65016126 (2002)

14. T. Galla, A.C.C. Coolen and D. Sherrington, preprint cond-mat/0303615 (2003)

15. A. De Martino, I. Giardina and G. Mosetti, preprint cond-mat/0305625 (2003)

16. L.F. Cugliandolo and J. Kurchan, J. Phys. A 275749 (1994)

17. A. Cavagna, J.-P. Garrahan, I. Giardina and D. Sherrington, Phys. Rev. Lett. 834429 (1999)

18. J.-P. Garrahan, E. Moro and D. Sherrington, Phys. Rev. E 62 R9 (2000)

19. M. Marsili and D. Challet, Phys. Rev. E 64056138 (2001)

20. D. Challet, M. Marsili and Y.-C. Zhang, Physica A 276 284 (2001) 